\title{
Por um planeamento urbano mais sustentável (parte II)
}

Quando pensámos na possibilidade de organizar um número temático da CIDADES, Comunidades e Territórios, dedicado ao tema do planeamento urbano e da sustentabilidade, estávamos longe de imaginar que, poucos meses depois, o mundo viveria uma pandemia, com impactos negativos imediatos, não só do ponto de vista de saúde pública, mas também do ponto de vista social, económico, cultural e político. Apesar das inevitáveis repercussões que estes impactos também tiveram no mundo académico e científico, mais de duas dezenas de submissões ao call do n. ${ }^{\circ} 41$ da Revista Cidades, justificaram a edição do presente Número Especial. Este número surge assim na continuidade da publicação de um Dossier temático, no n. ${ }^{\circ} 41$ da revista (dezembro de 2020), intitulado Por um planeamento urbano mais sustentável, e que contou com a apresentação de 10 textos, incluindo: 3 entrevistas, João Cunha Borges e Teresa Marat-Mendes a Francisco Silva Dias; Joana Carolina Schossler a Bernard Toulier; e Mariana Sanchez Salvador a Carolyn Steel; e 7 artigos científicos, da autoria de: Ricardo Cunha Dias, Diogo Guedes Vidal, Paulo Castro Seixas e Rui Leandro Maia; Rubens Amaral, Maria do Carmo de Lima Bezerra, Gustavo Macedo de Mello Baptista, Rômulo José da Costa Ribeiro e Aline da Nóbrega Oliveira; e Jasmine Pereira Vieira e Susana de Araújo Gastal; Teresa Marat-Mendes, João Cunha Borges, Sara Silva Lopes e Mafalda Matos Pereira; Jan Wolf, Paulo Batista e João Lourenço Marques; Ana Mélice Dias e Teresa Marat-Mendes; e Maria de Fátima Ferreiro, Isabel Salavisa e Sofia Bizarro.

No presente Número Especial incluem-se ao todo 13 textos, 2 entrevistas e 11 artigos científicos, submetidos ao escrutínio e avaliação científica de 2 ou mais revisores nacionais/internacionais, cujo trabalho, tempo e dedicação estamos naturalmente reconhecidas e gratas.

Duas entrevistas realizadas pelas editoras do presente Número Especial, Teresa Marat-Mendes e Patrícia Bento d'Almeida, abrem o Spring2021 da CIDADES, Comunidades e Territórios. A primeira entrevista, é dirigida ao Professor Catedrático Paulo Pinho, da Faculdade de Engenharia da Universidade do Porto, realizada no dia 30 de outubro de 2020. Paulo Pinho foi coordenador responsável do Projeto SPLACH - Spatial Planning for Change, um projeto de investigação que contou com a colaboração da Universidade do Porto, o Iscte - Instituto Universitário de Lisboa e a Universidade de Aveiro. Tratando-se de um projeto que procurou contribuir com futuras pistas para informar políticas publicas de planeamento urbano rumo a um futuro mais sustentável, a entrevista apresenta-nos um relato sobre o importante papel da investigação em planeamento urbano para alcançar a necessária evidencia científica. Apresentada de uma forma cronológica, a entrevista expõe como, em Portugal, importantes matérias societais, como por exemplo a luta contra a energia nuclear, foram coadjuvadas pela comunidade académica e científica nacional e internacional, e consequentemente determinantes também no âmbito das políticas publicas. O testemunho interpola problemáticas urbanas contemporâneas, nomeadamente no importante papel da ciência para a promoção da evidência científica e identificação das melhores soluções para a promoção de um Desenvolvimento Sustentável, bem como o próprio contributo do Projeto SPLACH para a reflecção necessária acerca do papel do planeamento urbano em Portugal, para o qual este projeto contribuiu.

A segunda entrevista foi dirigida ao arquiteto João Branco Pedro, desde 2019 Coordenador do Núcleo de Estudos Urbanos e Territoriais (NUT) do Laboratório Nacional de Engenharia Civil (LNEC). Realizada no dia 15 de outubro de 2020, a este arquiteto investigador foi solicitado que oferecesse um panorama breve da investigação sobre habitação desenvolvida no LNEC, particularmente sobre aquela que, desde 1994, esteve diretamente envolvido. Neste âmbito, ficou também sublinhada a ideia de que as linhas de investigação levadas a cabo no NUT, sempre procuraram dar resposta a problemas concretos e atuais da sociedade, nomeadamente nos domínios da construção, da habitação e urbanismo, do ambiente e da gestão dos riscos, apoiando deste modo o Estado na prossecução das políticas públicas. Foi ainda destacada pelo entrevistado a importância da "combinação entre a especialização e o diálogo interdisciplinar", favorecido naquele Laboratório pela presença diversificada de profissionais, com diferentes formações e especializações. Neste sentido, no panorama científico português, importa salientar o surgimento do estudo da arquitetura no LNEC - no início da década de 1960 - como uma ação

Copyright (C) 2021 (Marat-Mendes, T., Bento d'Almeida, P.) 
absolutamente visionária e pioneira naquele tempo, apenas anos mais tarde completada com a atividade científica desenvolvida em centros de investigação universitários.

No seguimento desta temática, o primeiro artigo científico que abre o presente Número Temático, elaborado por Patrícia Bento d'Almeida e Teresa Marat-Mendes, destaca a "A atualidade da investigação científica em arquitetura e urbanismo desenvolvida no LNEC (1961-1979) face ao desafio da sustentabilidade". As autoras revisitam ali alguns dos trabalhos de investigação desenvolvidos na Divisão de Construção e Habitação (19611969) do Serviço de Edifícios e Pontes e na subsequente Divisão de Arquitetura (1969-1979) do Serviço de Edifícios do LNEC, oferecendo uma leitura histórica colocada em perspetiva com as atuais problemáticas da sustentabilidade. Como referem as autoras, os mais recentes documentos publicados pelas Nações Unidas apontam para o repensar de temáticas em estudo no início dos anos 60, designadamente no LNEC. Inquéritos à habitação, ao espaço urbano e às condições de vida das populações outrora analisados, podem hoje ser retomados "para a análise da presente (e futura) problemática da sustentabilidade da vida urbana".

O artigo que se segue, "Drivers of change: how the food system of the Lisbon Metropolitan Area is being shaped by activities, initiatives and citizens needs towards a sustainable transition", foi elaborado por Teresa MaratMendes, Inês Isidoro, Joana Catela, Mafalda Pereira, João Borges, Sara Lopes e Carolina Henriques, no âmbito do Projeto SPLACH. Este artigo oferece-nos uma reflexão transdisciplinar sobre o impacto da espacialidade do sistema alimentar no território da Área Metropolitana de Lisboa, concluindo, acerca da pertinência deste sistema, numa revisão do processo de planeamento urbano. O artigo coloca em destaque como a realidade existente, modulada pelas atividades e iniciativas (no âmbito do sistema alimentar) da população que habita e trabalha sobre o território da Área Metropolitana de Lisboa, constitui um importante repositório de boas praticas que necessitam de ganhar maior escala e serem também elas acauteladas pelo planeamento urbano para a promoção de uma desejável transformação sustentável do território.

Também elaborado no âmbito do Projeto SPLACH, o artigo "Resiliência urbana pró-Sustentabilidade e planeamento sob incerteza”, sob a autoria de Carlos Gonçalves, Monique Borges e João Marques, oferece-nos um debate sobre formas de promover teorias e práticas de planeamento no sentido de responder ao crescente aumento de fontes de incerteza. Este artigo procura assim clarificar a constelação de conceitos e qual o estado de maturação do referencial teórico existente. Permite ainda considerar de que forma a resiliência pode servir de base normativa para a sustentabilidade e quais os pressupostos para planear resiliência urbana em contexto de ampliação de riscos, crises, catástrofes e incertezas.

Também dentro da temática da Sustentabilidade e do Planeamento Urbano, segue-se o artigo "Towards a necessary regenerative urban planning. Insights from community-led initiatives for ecocity transformation”, elaborado por Duncan Crowley, Teresa Marat-Mendes, Roberto Falanga, Thomas Henfrey e Gil Penha-Lopes. Este artigo sugere uma transição das atuais praticas de planeamento que, tal como no artigo "Drivers of change: how the food system of the Lisbon Metropolitan Area is being shaped by activities, initiatives and citizens needs towards a sustainable transition", realça o papel central das iniciativas locais no desenvolvimento sustentável e na própria revisão dos instrumentos de planeamento. Duncan Crowley et al, destacam o importante papel da participação dos cidadãos na regeneração dos seus territórios. Assim, procurando fazer a interligação entre ativismo e a academia, o artigo expõe os resultados da aplicação de um método de investigação/ação prática, de cariz transdisciplinar, desenvolvido para promover ecossistemas de 'Comunidades de Prática', e apoiar uma necessária transição socioeconómica e ambiental justa.

Segue-se o artigo "Disaster Preparedness Indicators: an application in the state of Paraná, Brazil", da autoria de Eduardo Gomes Pinheiro, do Corpo de Bombeiros do Paraná, e de Carlos Mello Garcias, Larissa Ferentz e Murilo Noli da Fonseca, da Pontifícia Universidade Católica do Paraná. Trata-se de uma análise que tem por objetivo avaliar a capacidade de prevenção e mitigação de riscos dos municípios para a ocorrência de desastres naturais, tais como tempestades, inundações, terremotos, maremotos, vulcões, etc... Como referido pelos autores, o Brasil está entre os países do mundo mais afetados por desastres naturais ou tecnológicos, assim, foram selecionados dez municípios do estado do Paraná como casos de estudo. Como conclusões da análise efetuada, os autores apontam o facto de os municípios analisados não preverem, nos seus planos de contingência, áreas de risco rural ou desastre marítimo. Por último, chamam ainda a atenção para a importância do instrumento de análise por eles aplicado - 
“principalmente se estiver inserido no Sistema Estadual de Defesa Civil (SISDC)" -, para uma análise do existente face a melhores políticas de Defesa Civil.

Transportando-nos agora para uma realidade sul americana, no Brasil, Tobias Kühner, Carlos Vinícius da Silva Pinto e Cláudia Naves David Amorim, através do artigo "International urban agendas and sustainable integrated urban development in developing countries: the case of Brazil", expõem um retrato de como as agendas internacionais, nomeadamente a Nova Agenda Urbana, enquadram as grandes transformações urbanas que estão a ocorrer na contemporaneidade. Consequentemente, os autores oferecem um panorama do contexto da implementação de agendas urbanas e suas definições de sustentabilidade. Examinam ainda como determinados documentos doutrinários, não vinculantes a Nova Agenda Urbana, devem ser implementados às escalas regional e nacional. A definição das agendas urbanas sustentáveis e os seus desafios internacionais comuns são desconstruídos pelos autores e comparados com os princípios estabelecidos pela Nova Agenda Urbana.

$\mathrm{O}$ artigo "A sustentabilidade no território difuso: reflexões sobre o caminho a seguir", da autoria de uma investigadora do Centro de Estudos de Geografia e Ordenamento do Território, Catarina de Almeida Pinheiro, depois de uma breve contextualização da mediatização da sustentabilidade - desde a publicação do relatório de Brundtland, em 1987 -, a autora oferece-nos uma caracterização do território da urbanização difusa do Baixo Minho, comparando-o (metaforicamente) com um palimpsesto, pelo facto deste ter sido constantemente alterado por fruto da humanização "mas sem rutura evidente entre a cidade e o campo". Face ao exposto, apesar da política de Ordenamento do Território português ser definida pela Lei de bases gerais da política pública de solos, de ordenamento do território e de urbanismo $(2014,2017)$, a autora defende ali impor-se "a promoção de uma 'cultura do território" para podermos observar um (futuro) território mais sustentável e resiliente. Ao longo da análise efetuada descrevem-se possíveis estratégias para uma gestão sustentável do solo.

Segue-se o artigo "Ação pública para redução da vacância imobiliária: a implementação de instrumentos da função social da propriedade na cidade de São Paulo”, da autoria de Camila Nastari Fernandes e de Marcela Alonso Ferreira. As autoras apresentam primeiramente, a partir de uma perspetiva histórica, a trajetória do planeamento urbano da cidade de São Paulo, no Brasil, muito "voltada às ações de controle sobre uso e ocupação do solo urbano". Face à situação de carência habitacional verificada, defendem o combate à "vacância imobiliária" desocupação de imóveis ou ao mau aproveitamento destes -, com vista ao cumprimento da "função social da propriedade", cuja implementação dos seus instrumentos surge durante a prefeitura de Fernando Haddad (20132017). A análise efetuada, balizada entre os anos 2014-2016, permitiu constatar que a aplicação destes instrumentos reduz a vacância imobiliária, sendo por este motivo, não só de referência para outros municípios brasileiros, mas, principalmente, fundamental para avaliar a política de gestão e transformação do solo urbano.

No artigo "Por um urbanismo sustentável: Um olhar sobre a vivência nas centralidades de subúrbios cariocas", da autoria de uma investigadora da Universidade Federal do Rio de Janeiro, Maria Paula Albernaz apresenta uma reflexão sobre os limites dos instrumentos de ordenamento territorial atuais e a necessidade de políticas públicas para intervirem nas centralidades suburbanas. A autora analisa os territórios suburbanos da região metropolitana da cidade do Rio de Janeiro, no Brasil, com vista a refletir sobre a noção de centralidade e de planeamento urbano, expondo eventuais potencialidades para a desejável sustentabilidade urbana. Como conclusão da análise efetuada, uma nova perspetiva é oferecida pela autora: para um urbanismo mais sustentável "há que também levar em conta seus atributos urbanísticos e paisagísticos provenientes de sua trama original que oferece ambientes de conforto e latente sociabilidade ao espaço urbano, e a adaptabilidade que o grão pequeno pode oferecer a múltiplas e constantes demandas da população local”.

Brendow de Oliveira Fraga, Magnus Emmendoerfer, Vânia Gonçalves Costa e Alcielis de Paula Neto são autores do texto que se segue "Em direção ao movimento Slow City: planejamento público coletivo na sustentabilidade de um pequeno município brasileiro”. Partindo do pressuposto do movimento 'Slow City' os autores defendem esta teoria como uma possibilidade para a mitigação dos problemas socio ambientais contemporâneos. O artigo expõe ainda um estudo dedicado à gestão do território de forma compartilhada como proposta promissora de sustentabilidade, aplicado num território específico do Brasil. O objetivo é analisar o processo de planeamento para a mobilização dos diferentes atores sociais de um pequeno município brasileiro para os fins de qualificá-lo na filosofia Slow City. Tal como nos artigos "Towards a necessary regenerative urban planning. Insights from community-led initiatives for ecocity transformation", de Duncan Crowley et all., e "Drivers of change: how the 
food system of the Lisbon Metropolitan Area is being shaped by activities, initiatives and citizens needs towards a sustainable transition", de Marat-Mendes et all., o artigo de Fraga et al. reflete sobre o importante papel do envolvimento dos cidadãos para alcançar um melhor desenvolvimento urbano. Um papel que fornecerá mais facilmente a identificação das informações necessárias para a elaboração de diagnósticos locais e de propostas de ações que necessitam de serem asseguradas.

Por último, o artigo "Planning the sustainable development of historic neighbourhoods through the management of significance: A proposal for a values-based approach in Santarém's Mouraria” de Matthew Nouch propõe um olhar atento por parte do planeamento urbano sobre as áreas históricas, no sentido de as integrar nas suas agendas urbanas e regionais, considerando-as como parte integrante de uma unidade que não apenas a classificada como património histórico. O autor chama a atenção para a urgente necessidade de se contrariarem as atuais políticas de fossilização do património e de, em vez disso, o potencializar como parte integrante do processo de regeneração urbana necessária ao desenvolvimento urbano. Aplicado ao bairro da Mouraria em Santarém, em Portugal, o autor apresenta os resultados de um estudo em que defende a operacionalização das recomendações da abordagem da Paisagem Urbana Histórica (HUL), no sentido de produzir evidencias robustas que coproduzidas com as comunidades locais, poderão apoiar políticas de planeamento urbano sustentáveis, consistentes com a legislação urbana e de conservação da União Europeia e nacional.

Teresa Marat-Mendes, Professora Associada com Agregação no Departamento de Arquitectura e Urbanismo do Iscte e Investigadora integrada no DINÂMIA'CET-Iscte.

Patrícia Bento d'Almeida, Investigadora integrada no DINÂMIA'CET-Iscte.

Editoras do Número Especial

\section{Financiamento}

O presente dossier temático surge no âmbito do projeto de investigação "SPLACH - Spatial Planning for Change" (POCI-01-0145-FEDER-016431), coordenado no DINÂMIA'CET-Iscte por Teresa Marat-Mendes com financiamento de Fundos Europeus Estruturais e de Investimento (FEEI) através do Programa Operacional Competitividade e Internacionalização (COMPETE 2020) na sua componente FEDER, e por Fundos Nacionais através da Fundação para a Ciência e a Tecnologia na sua componente OE.

Parte da investigação conduzida para a elaboração do presente dossier temático é também oriunda do projeto de pós-doutoramento "O LNEC e a História da Investigação em Arquitectura" (SFRH/BPD/117167/2016), desenvolvido por Patrícia Bento d'Almeida, e financiado pela Fundação para a Ciência e a Tecnologia através do programa de financiamento FSE.

\section{Agradecimentos}

As Editoras do Número Especial agradecem à equipa editorial da revista CIDADES, Comunidades e Territórios, particularmente a Maria Assunção Gato, Ana Rita Cruz e Mariana Leite Braga, pelo suporte dado ao longo de todo o processo.

As Editoras do Dossier agradecem ainda a todos os revisores*, especialistas independentes que, de acordo com o modelo de revisão de pares da revista, avaliaram e escrutinaram cuidadosamente cada um dos artigos submetidos, promovendo a publicação de duas edições temáticas de qualidade - Dossier temático $\left(\mathrm{N}^{\circ} 41\right)$ e presente Número Especial (Sp21). 
* Alberto Cuchi (Universidade Politécnica da Catalunha); Ana Claudia Cardoso (Universidade Federal do Pará); Ana Patrícia Oliveira (Instituto Superior Técnico); Ana Rita Cruz (Iscte-Instituto Universitário de Lisboa); Andreia Savedra Cardoso (Instituto Superior de Agronomia da Universidade de Lisboa); Álvaro Domingos (Faculdade de Arquitetura da Universidade do Porto); Agustín Hernández Aja (Escuela Técnica Superior de Arquitectura Universidad Politécnica de Madrid); Artur Rosa Pires (Universidade de Aveiro); Carlos Nunes Silva (Instituto de Geografia e Ordenamento do Território da Universidade de Lisboa); Catarina Camarinhas (Faculdade de Arquitetura da Universidade de Lisboa); Cecília Delgado (Universidade NOVA de Lisboa); Colin Moorcraft; Cristina Soares Ribeiro Gomes Cavaco (Faculdade de Arquitetura da Universidade de Lisboa); David Leite Viana (Iscte-Instituto Universitário de Lisboa); Eduarda Vieira (Universidade Católica Portuguesa); Elisabete Tomaz (Iscte-Instituto Universitário de Lisboa); Esmeralda Paupério (Faculdade de Engenharia da Universidade do Porto); Eulalia Gomez-Escoda (Universitat Politècnica de Catalunya); Fernando Nunes da Silva (Instituto Superior Técnico); Filipa Serpa (Faculdade de Arquitetura da Universidade de Lisboa); Heraldo Borges (Universidade Presbiteriana Mackenzie); Humberto Yamaki (Universidade Estadual de Londrina); Inês Isidoro (Iscte-Instituto Universitário de Lisboa); Ioannis Poulios (Ahmedabad University); Isabel Guerra (Iscte-Instituto Universitário de Lisboa); Joana Mourão (Instituto Superior Técnico); João Cabral (Faculdade de Arquitetura da Universidade de Lisboa); João Carlos Azevedo (Instituto Politécnico de Bragança); João Ferrão (Instituto de Ciências Sociais); José Alberto Vieira Rio Fernandes (Faculdade de Letras da Universidade do Porto); José Manuel Henriques (IscteInstituto Universitário de Lisboa); Luísa Batista (Faculdade de Engenharia da Universidade do Porto); Małgorzata Hanzl (Technical University of Lodz); Manuel Pacheco Coelho (Instituto Superior de Economia e Gestão da Universidade de Lisboa); Maria da Graça Moreira (Faculdade de Arquitetura da Universidade de Lisboa); Margarida Perestrelo (Iscte-Instituto Universitário de Lisboa); Mayté Banzo (Université Bordeaux Montaigne); Miguel Sopas de Melo Bandeira (Universidade do Minho); Obas John Ebohon (London South Bank University); Paul Osmond (University of New South Wales Sidney); Paulo Pinho (Faculdade de Engenharia da Universidade do Porto); Pedro Costa (Iscte-Instituto Universitário de Lisboa); Pedro George (Faculdade de Arquitectura da Universidade de Lisboa); Renato Leão Rego (Universidade Estadual de Maringá); Rita Ochoa (Iscte-Instituto Universitário de Lisboa); Roberto Falanga (Instituto de Ciências Sociais); Rui Jorge Garcia Ramos (Faculdade de Arquitetura da Universidade do Porto); Silvia Tavares (University of the Sunshine Coast); Stefania Stellacci (IscteInstituto Universitário de Lisboa); Susan Parham (University of Hertfordshire); Tiago Brandão (Universidade NOVA de Lisboa); Yves Cabannes (University College London); Valério Augusto Soares de Medeiro (Faculdade de Arquitetura e Urbanismo da Universidade de Brasília); Vera Regina Tângari (Universidade Federal do Rio de Janeiro); Walter Rodrigues (Iscte-Instituto Universitário de Lisboa). 